FACTA UNIVERSITATIS (NIŠ)

Ser. Math. Inform. Vol. 35, No 2 (2020), 485-493

https://doi.org/10.22190/FUMI2002485Y

\title{
SOME NEW TYPES OF CONTINUITY IN ASYMMETRIC METRIC SPACES
}

\author{
Taja Yaying, Bipan Hazarika and Syed Abdul Mohiuddine*
}

(C) 2020 by University of Niš, Serbia | Creative Commons Licence: CC BY-NC-ND

\begin{abstract}
Using the notions of forward and backward arithmetic convergence in asymmetric metric spaces, we have defined arithmetic $f f$-continuity and arithmetic $f b$ continuity and prove some interesting results. Moreover, we have introduced the concepts of forward and backward arithmetic compactness and obtained the related results in the setting of asymmetric metric space.

Keywords: asymmetric metric spaces; forward and backward arithmetic compactness; forward and backward arithmetic convergence; arithmetic $f f$-continuity.
\end{abstract}

\section{Introduction and Preliminaries}

In 1931, Wilson [18] first introduced asymmetric metric spaces as quasi-metric spaces, and afterwards they were studied by many other authors (see $[1,14,15,16]$ ). An asymmetric metric space is a generalization of a metric space but the symmetry axiom is eliminated in the definition of metric spaces. We can come up with some troubles in several classical statements of symmetric analysis without the symmetry property in the definition of such spaces. In asymmetric metric spaces, some notions such as convergence, completeness and compactness are different from the metric case. There are two notions for each of them, namely forward and backward ones, since we have two topologies which are the forward topology and the backward topology in the same space (see [13]). Collins and Zimmer [10] studied these notions in the asymmetric context.

An example that asymmetric metrics are common in real life is taxicab geometry topology including one-way streets, where a path from point $A$ to point $B$ contains a different set of streets than a path from $B$ to $A$. Also, the examples of the latest applications of asymmetric metric spaces in the field of pure and applied

Received April 13, 2019; accepted September 12, 2019

2010 Mathematics Subject Classification. Primary 40A35; Secondary 40A05, 26A15

* Corresponding author 
mathematics and material science are as in [8]. In [9], Cobzas gave the basic results on asymmetric normed spaces.

Ruckle [17] introduced the notion ofarithmetic convergence as a sequence $x=$ $\left(x_{k}\right)$ defined on $\mathbb{N}$, and it is said to be arithmetic convergent if for each $\varepsilon>0$ there is an integer $n$ such that for every integer $m$ we have $\left|x_{m}-x_{<m, n>}\right|<\varepsilon$. Here and henceforth, $\langle m, n\rangle$ denotes the greatest common divisor of $m$ and $n$. Çakalli [4] gave another definition of arithmetic convergence of a sequence $\left(x_{k}\right)$ as a sequence $x=\left(x_{k}\right)$ is said to be arithmetically convergent if for each $\varepsilon>0$ there is an integer $n_{0}$ such that $\left|x_{m}-x_{<m, n>}\right|<\varepsilon$ for every integers $m, n$ satisfying $<m, n>\geq n_{0}$. Throughout the article, we follow the definition given by Çakalli in his corrigendum to the paper [4]. For more details on arithmetic convergence and arithmetic continuity, we refer to [4, 19, 20, 21, 22, 23]. For different types of continuity and $b$ - metric spaces, we refer to $[2,3,5,6,7,11,12]$.

In this article, we will first introduce the concepts of forward and backward arithmetic convergence and using these notions we will define forward and backward arithmetic continuity in asymmetric metric spaces and establish some interesting results. In the last section, we will introduce forward and backward arithmetic compactness and obtain related results.

\section{Asymmetric Metric Spaces}

Let us recall some definitions and results on asymmetric metric spaces which were given in [10].

Definition 2.1. A function $d: X \times X \rightarrow R$ is an asymmetric metric and $(X, d)$ is an asymmetric metric space if

(i) $d(x, y) \geq 0$ and $d(x, y)=0$ holds if and only if $x=y$ for every $x, y \in X$.

(ii) $d(x, z) \leq d(x, y)+d(y, z)$; for every $x, y, z \in X$.

Definition 2.2. The forward topology $\tau_{+}$induced by $d$ is the topology generated by the forward open balls $B^{+}(x, \varepsilon)=\{y \in X: d(x, y)<\varepsilon\}$ for $x \in X ; \varepsilon>0$.

Likewise, the backward topology $\tau_{-}$induced by $d$ is the topology generated by the backward open balls $B^{-}(x, \varepsilon)=\{y \in X: d(y, x)<\varepsilon\}$ for $x \in X ; \varepsilon>0$.

Definition 2.3. A set $S \subset X$ is forward bounded (resp. backward bounded), if there exists $x \in X$ and $\varepsilon>0$ such that $S \subset B^{+}(x, \varepsilon)$ (resp. $S \subset B^{-}(x, \varepsilon)$ ).

Definition 2.4. A sequence $\left(x_{n}\right)$ is said to be forward convergent to $x \in X$ (backward convergent to $x \in X)$ if and only if

$$
\lim _{n \rightarrow \infty} d\left(x, x_{n}\right)=0\left(\lim _{n \rightarrow \infty} d\left(x_{n}, x\right)=0\right)
$$

and is denoted by $x_{n} \stackrel{f}{\rightarrow} x\left(x_{n} \stackrel{b}{\rightarrow} x\right)$. 
Definition 2.5. A sequence $\left(x_{n}\right)$ in an asymmetric metric space $(X, d)$ is forward Cauchy (backward Cauchy) if for each $\varepsilon>0$ there exists a $N \in \mathbb{N}$ such that for $k \geqslant n \geqslant N ; d\left(x_{n}, x_{k}\right)<\varepsilon\left(d\left(x_{k}, x_{n}\right)<\varepsilon\right)$ holds.

Definition 2.6. [Sequential definition of continuity] Let $\left(X, d_{X}\right)$ and $\left(Y, d_{Y}\right)$ be asymmetric metric spaces. A function $f: X \rightarrow Y$ is $f f-$ continuous at $x \in X$ if and only if whenever $x_{n} \stackrel{f}{\rightarrow} x$ in $\left(X, d_{X}\right)$ we have $f\left(x_{n}\right) \stackrel{f}{\rightarrow} f(x)$ in $\left(Y, d_{Y}\right)$.

The statement holds analogously for the other types. Note that the forward uniform continuity is same as the backward uniform continuity.

Definition 2.7. A set $S \subset X$ is

(i) forward compact if every open cover of $S$ in the forward topology has a finite subcover.

(ii) forward relatively compact if $\bar{S}$ is forward compact, where $\bar{S}$ denotes the closure of $S$ in the forward topology.

(iii) forward sequentially compact if every sequence in $X$ contains a forward convergent subsequence.

(iv) forward complete if every forward Cauchy sequence is forward convergent.

Definition 2.8. Let $\left(f_{n}\right)$ be a function sequence and $f$ be a function from $X$ to $Y$. We say that the sequence $\left(f_{n}\right)$ is forward convergent uniformly (backward convergent uniformly) to limit $f$ if for every $\varepsilon>0$ there exists a positive number $N$ such that for all $x \in X$ and all $n \geq N$ we have $d\left(f(x), f_{n}(x)\right)<\varepsilon\left(d\left(f_{n}(x), f(x)\right)<\varepsilon\right)$.

\section{Arithmetic Continuity in Asymmetric Metric Spaces}

In this section, we introduce the concepts of forward and backward arithmetic convergence and forward and backward arithmetic continuity in asymmetric metric spaces and prove some results using these notions.

Definition 3.1. A sequence $x=\left(x_{k}\right)$ is called forward arithmetic convergent (resp. backward arithmetic convergent) in an asymmetric metric space $(X, d)$ if for each $\varepsilon>0$ there is an integer $N$ such that $d\left(x_{<m, n>}, x_{m}\right)<$ $\varepsilon$ (resp. $d\left(x_{m}, x_{<m, n>}\right)<\varepsilon$ ), for every integers $m, n$ satisfying $<m, n>\geq N$. We shall denote it by writing $x_{m} \stackrel{a f}{\rightarrow} x_{<m, n>}$ (resp. $\left.x_{m} \stackrel{a b}{\rightarrow} x_{<m, n>}\right)$.

Definition 3.2. Let $\left(X, d_{X}\right)$ and $\left(Y, d_{Y}\right)$ be two asymmetric metric spaces. A function $f: X \rightarrow Y$ is arithmetic $f f$ - continuous (respectively arithmetic $f b-$ continuous $)$, iff it transforms forward arithmetic convergent sequence in $\left(X, d_{X}\right)$ to forward arithmetic convergent sequence (respectively backward arithmetic convergent sequence) in $\left(Y, d_{Y}\right)$. 
Theorem 3.1. Let $\left(X, d_{X}\right)$ and $\left(Y, d_{Y}\right)$ be asymmetric metric spaces. If $f: X \rightarrow$ $Y$ is uniformly continuous then it is arithmetic $f f$-continuous.

Proof. Let $f: X \rightarrow Y$ be uniformly continuous and $\left(x_{n}\right)$ be any forward arithmetic convergence sequence in $X$. Since $f$ is uniformly continuous, for a given $\varepsilon>0$ there exists $\delta>0$ such that for every $x, y \in X$ with $d_{X}(x, y)<\delta, d_{Y}(f(x), f(y))<\varepsilon$.

Again, the sequence $\left(x_{n}\right)$ is forward arithmetic convergent in $X$, hence for the same $\delta>0$ there exists a positive integer $m_{0}$ such that for all integers $m, n$ satisfying $<m, n>\geq 0$,

$$
\begin{aligned}
d_{X}\left(x_{<n, m>}, x_{n}\right)<\delta \text { for each } n & \Rightarrow d_{Y}\left(f\left(x_{<n, m>}\right), f\left(x_{n}\right)\right)<\varepsilon \text { for each } n \\
& \Rightarrow \text { the sequence }\left(f\left(x_{n}\right)\right) \text { is forward arithmetic } \\
& \text { convergent } \\
& \Rightarrow \text { the function } f \text { is arithmetic ff-continuous. }
\end{aligned}
$$

This completes the proof.

Definition 3.3. A sequence of functions $\left(f_{n}\right)$ from an asymmetric metric space $\left(X, d_{X}\right)$ to an asymmetric metric space $\left(Y, d_{Y}\right)$ is said to be forward arithmetic convergent (resp. backward arithmetic convergent) if for any $\varepsilon>0$ and $\forall x \in X$ there exists a positive integer $m_{0}$ such that for all integers $m, n$ satisfying $\langle m, n\rangle \geq$ 0 ,

$$
d_{Y}\left(f_{<n, m>}(x), f_{n}(x)\right)<\varepsilon\left(\text { resp. } d_{Y}\left(f_{n}(x), f_{<n, m>}(x)\right)<\varepsilon\right) .
$$

Theorem 3.2. If $\left(f_{n}\right)$ be a sequence of forward arithmetic convergent functions from an asymmetric metric space $\left(X, d_{X}\right)$ to an asymmetric metric space $\left(Y, d_{Y}\right)$ and $x_{o}$ is a point in $X$ such that

$$
\lim _{x \rightarrow x_{o}} f_{n}(x)=y_{n}, n=1,2,3 \ldots
$$

then $\left(y_{n}\right)$ is also forward arithmetic convergent.

Proof. Since the sequence $\left(f_{n}\right)$ is forward arithmetic convergent, therefore, for $\varepsilon>0$ and a positive integer $m_{0}$ such that for all integers $m, n$ satisfying $\langle m, n\rangle \geq 0$

$$
d_{Y}\left(f_{<n, m>}(x), f_{n}(x)\right)<\varepsilon \forall x \in X .
$$

Keeping $n, m$ fixed and letting $x \rightarrow x_{o}$,

$$
d_{Y}\left(y_{<n, m>}, y_{n}\right)<\varepsilon .
$$

Hence, the sequence $\left(y_{n}\right)$ is forward arithmetic convergent.

Remark 3.1. The same result can be written for backward arithmetic convergence. 
Theorem 3.3. If $\left(f_{n}\right)$ is a sequence of arithmetic $f f$-continuous functions from asymmetric metric space $\left(X, d_{X}\right)$ to asymmetric metric space $\left(Y, d_{Y}\right)$ with forward convergence equivalent to backward convergence in $Y$ and $\left(f_{n}\right)$ forward converges uniformly to a function $f$, then $f$ is arithmetic $f f$-continuous.

Proof. Let $\varepsilon>0$ and $\left(x_{n}\right)$ be any forward arithmetic convergent sequence in $X$. Since $f_{n} \stackrel{f}{\rightarrow} f$ uniformly, we can choose $N_{1} \in \mathbb{N}$ so that $d_{Y}\left(f(x), f_{n}(x)\right)<\frac{\varepsilon}{3}$ for all $n \geq N_{1}$ and $x \in X$. Now, in particular, $f_{n}\left(x_{<n, m>}\right) \stackrel{f}{\rightarrow} f\left(x_{<n, m>}\right)$ and so $f_{n}\left(x_{<n, m>}\right) \stackrel{b}{\rightarrow} f\left(x_{<n, m>}\right)$. Thus, we can find $N_{2} \in \mathbb{N}$ so that $d_{Y}\left(f_{n}\left(x_{<n, m>}\right), f\left(x_{<n, m>}\right)\right)<\frac{\varepsilon}{3}$ for all $n \geq N_{2}$. Let $N=\max \left\{N_{1}, N_{2}\right\}$. Further, $\left(f_{n}\right)$ is given to be a sequence of arithmetic $f f$-continuous functions. In particular, $f_{N}$ is arithmetic $f f$-continuous function, and thus arithmetic $f b$-continuous by equivalence of forward and backward convergence in $Y$. So there exists an integer $n_{0}$, greater than $N$ and $\delta>0$ such that

$$
d_{Y}\left(f_{N}\left(x_{n}\right), f_{N}\left(x_{<n, m>}\right)\right)<\frac{\varepsilon}{3} \text { for } d_{X}\left(x_{<n, m>}, x_{n}\right)<\delta,
$$

for all integers $m, n$ satisfying $<m, n>\geq n_{0}$. Consequently, whenever $d_{X}\left(x_{<n, m>}, x_{n}\right)<\delta$ and $<m, n>\geq n_{0}$, we have

$$
\begin{aligned}
d_{Y}\left(f\left(x_{n}\right), f\left(x_{<n, m>}\right)\right) \leq & d_{Y}\left(f\left(x_{n}\right), f_{N}\left(x_{n}\right)\right)+d_{Y}\left(f_{N}\left(x_{n}\right), f_{N}\left(x_{<n, m>}\right)\right) \\
& +d_{Y}\left(f_{N}\left(x_{<n, m>}\right), f\left(x_{<n, m>}\right)\right) \\
< & \frac{\varepsilon}{3}+\frac{\varepsilon}{3}+\frac{\varepsilon}{3}=\varepsilon .
\end{aligned}
$$

Therefore $f$ is arithmetic $f b$-continuous and by equivalence of convergence it is also arithmetic $f f$-continuous.

Theorem 3.4. Let $\left(X, d_{X}\right)$ and $\left(Y, d_{Y}\right)$ be two asymmetric metric spaces. Then the set of all arithmetic $f f$-continuous functions from $X$ to $Y$, with forward convergence equivalent to backward convergence in $Y$, is a closed subset of all continuous functions from $X$ to $Y$ i.e. $\mathbb{A}^{f f}(X, Y)=\overline{\mathbb{A}^{f f}(X, Y)}$ where $\mathbb{A}^{f f}(X, Y)$ is the set of all arithmetic $f f$-continuous functions from $X$ to $Y$ and $\overline{\mathbb{A}^{f f}(X, Y)}$ denotes the closure of $\mathbb{A}^{f f}(X, Y)$.

Proof. Let $f \in \overline{\mathbb{A}^{f f}(X, Y)}$. Then there exists a sequence of points in $\mathbb{A}^{f f}(X, Y)$ such that $f_{n} \stackrel{f}{\rightarrow} f$ as $n \rightarrow \infty$. Let $\varepsilon>0$ and $\left(x_{n}\right)$ be any forward arithmetic convergent sequence in $X$. Since $f_{n} \stackrel{f}{\rightarrow} f$ uniformly, we can choose $N_{1} \in \mathbb{N}$ so that $d_{Y}\left(f(x), f_{n}(x)\right)<\frac{\varepsilon}{3}$ for all $n \geq N_{1}$ and $x \in X$. In particular, $f_{n}\left(x_{<n, m>}\right) \stackrel{f}{\rightarrow}$ $f\left(x_{<n, m>}\right)$ and so $f_{n}\left(x_{<n, m>}\right) \stackrel{b}{\rightarrow} f\left(x_{<n, m>}\right)$. Therefore, we can find $N_{2} \in \mathbb{N}$ so that $d_{Y}\left(f_{n}\left(x_{<n, m>}\right), f\left(x_{<n, m>}\right)\right)<\frac{\varepsilon}{3}$ for all $n \geq N_{2}$. Assume that $N=\max \left\{N_{1}, N_{2}\right\}$. Moreover, $\left(f_{n}\right)$ is given to be a sequence of arithmetic $f f$-continuous functions. 
In particular, $f_{N}$ is arithmetic $f f$-continuous function, and thus arithmetic $f b$ continuous by equivalence of forward and backward convergence in $Y$. So there exists an integer $n_{0}$ greater than $N$ and $\delta>0$ such that

$$
d_{Y}\left(f_{N}\left(x_{n}\right), f_{N}\left(x_{<n, m>}\right)\right)<\frac{\varepsilon}{3} \text { for } d_{X}\left(x_{<n, m>}, x_{n}\right)<\delta
$$

for all integers $m, n$ satisfying $<m, n>\geq n_{0}$. Thus, whenever $d_{X}\left(x_{<n, m>}, x_{n}\right)<\delta$ and $\langle m, n\rangle \geq n_{0}$, we have

$$
\begin{aligned}
d_{Y}\left(f\left(x_{n}\right), f\left(x_{<n, m>}\right)\right) \leq & d_{Y}\left(f\left(x_{n}\right), f_{N}\left(x_{n}\right)\right)+d_{Y}\left(f_{N}\left(x_{n}\right), f_{N}\left(x_{<n, m>}\right)\right) \\
& +d_{Y}\left(f_{N}\left(x_{<n, m>}\right), f\left(x_{<n, m>}\right)\right) \\
< & \frac{\varepsilon}{3}+\frac{\varepsilon}{3}+\frac{\varepsilon}{3}=\varepsilon .
\end{aligned}
$$

Therefore $f$ is arithmetic $f b$-continuous and by equivalence of convergence it is also arithmetic $f f$-continuous. So $f \in \mathbb{A}^{f f}(X, Y)$. This completes the prove of the theorem.

In [4], Çakalli introduced the notion of $(c A C)$-continuity as follows: a function $f$ is said to be $(c A C)$-continuous (or $f \in(c A C)$ ) if $f$ transforms convergent sequences to arithmetic convergent sequences. We define this notion in the sense of arithmetic forward (or backward) convergence as follows:

A function $f$ from asymmetric metric space $X$ to asymmetric metric space $Y$ is said to be forward $(c A C)$-continuous if it transforms forward convergent sequences in $X$ to forward arithmetic convergent sequences in $Y$, i.e. $\left(x_{n}\right)$ is forward convergent in $X$ implies $f\left(x_{n}\right)$ is forward arithmetic convergent in $Y$.

Theorem 3.5. Let $\left(X, d_{X}\right)$ and $\left(Y, d_{Y}\right)$ be two asymmetric metric spaces, with forward convergence equivalent to backward convergence in $Y$. If $\left(f_{n}\right)$ is a sequence of forward $(c A C)$-continuous functions from $X$ to $Y$ and $\left(f_{n}\right)$ forward converges uniformly to a function $f$, then $f$ is forward $(c A C)$-continuous.

Proof. Let $\varepsilon>0$ be given and $\left(x_{k}\right)$ be any forward convergent sequence in $X$. Since $f_{n}$ forward converges uniformly to $f$, there exists a positive integer $N_{1}$ such that $d_{Y}\left(f(x), f_{n}(x)\right)<\frac{\varepsilon}{3}$ for all $x \in X$ and $n \geq N_{1}$. In particular, $f_{n}\left(x_{n}\right) \stackrel{f}{\rightarrow} f\left(x_{n}\right)$ and so $f_{n}\left(x_{n}\right) \stackrel{b}{\rightarrow} f\left(x_{n}\right)$. Thus we can find $N_{2} \in \mathbb{N}$ so that $d_{Y}\left(f_{n}\left(x_{n}\right), f\left(x_{n}\right)\right)<\frac{\varepsilon}{3}$ for all $n \geq N_{2}$. Assume that $N=\max \left\{N_{1}, N_{2}\right\}$. By hypothesis, $f_{n}$ is forward $(c A C)$-continuous. In particular $f_{N}$ is forward $(c A C)$-continuous, so there exists an integer $n_{0}$, greater than $N$ such that $d_{Y}\left(f_{N}\left(x_{<m, n>}\right), f_{N}\left(x_{n}\right)\right)<\frac{\varepsilon}{3}$ for all $x \in X$ and for all integers $m, n$ satisfying $\langle m, n\rangle \geq n_{0}$. Thus, it follows that

$$
\begin{aligned}
d_{Y}\left(f\left(x_{<m, n>}\right), f\left(x_{n}\right)\right) \leq & d_{Y}\left(f\left(x_{<m, n>}\right), f_{N}\left(x_{<m, n>}\right)\right) \\
& +d_{Y}\left(f_{N}\left(x_{<m, n>}\right), f_{N}\left(x_{n}\right)\right)+d_{Y}\left(f_{N}\left(x_{n}\right), f\left(x_{n}\right)\right) \\
< & \frac{\varepsilon}{3}+\frac{\varepsilon}{3}+\frac{\varepsilon}{3}=\varepsilon .
\end{aligned}
$$

This establishes the result. 
Theorem 3.6. Let $\left(X, d_{X}\right)$ and $\left(Y, d_{Y}\right)$ be two asymmetric metric spaces. Then the set of all forward $(c A C)$-continuous functions from $X$ to $Y$, with forward convergence equivalent to backward convergence in $Y$, is a closed subset of the set of all continuous functions from $X$ to $Y$.

Proof. The result immediately follows from the previous theorem.

\section{Compactness in Asymmetric Metric Spaces}

We will first introduce forward arithmetic compactness and backward arithmetic compactness in the setting of asymmetric metric space as follows:

Definition 4.1. A subset $A$ of an asymmetric metric space $\left(X, d_{X}\right)$ is said to be

(i) forward arithmetic compact if every sequence in $A$ has forward arithmetic convergent subsequence.

(ii) backward arithmetic compact if every sequence in $A$ has backward arithmetic convergent subsequence.

Theorem 4.1. An arithmetic $f f$-continuous image of an forward arithmetic compact subset of an asymmetric metric space $(X, d)$ is forward arithmetic compact.

Proof. Let $\left(X, d_{X}\right)$ and $\left(Y, d_{Y}\right)$ be asymmetric metric spaces. Let $f: X \rightarrow Y$ be an arithmetic $f f$-continuous function and $A \subset X$ be forward arithmetic compact. Let $\left(y_{n}\right)$ be a sequence in $f(A)$. Then we can write $y_{n}=f\left(x_{n}\right)$ where $x_{n} \in X$ for each $n \in \mathbb{N}$. Since $A$ is forward arithmetic compact, there exists an forward arithmetic convergent subsequence $\left(x_{n_{k}}\right)$ of $\left(x_{n}\right)$. Again, it is given that $f$ is arithmetic $f f$ continuous, this implies that $f\left(x_{n_{k}}\right)$ is forward arithmetic convergent subsequence of $f\left(x_{n}\right)$. Hence, $f(A)$ is forward arithmetic compact.

Theorem 4.2. An arithmetic fb-continuous image of a backward arithmetic compact subset of an asymmetric metric space $(X, d)$ is backward arithmetic compact.

Proof. The proof is the same as in the previous theorem.

Theorem 4.3. Any closed subset of a forward arithmetic compact subset of an asymmetric metric space $(X, d)$ is forward arithmetic compact.

Proof. Let $A$ be any forward arithmetic compact subset of $X$ and $B$ be a closed subset of $A$. Let $x=\left(x_{n}\right)$ be any sequence of points in $B$. Then $x=\left(x_{n}\right)$ is a sequence of points in $A$. Since $A$ is forward arithmetic compact, there exists an forward arithmetic convergent subsequence $\left(x_{n_{k}}\right)$ of the sequence $x$. Since $B$ is closed, so any sequence $x=\left(x_{n}\right)$ of points in $B$ has forward arithmetic convergent subsequence in $B$. Hence the result. 
Theorem 4.4. Any closed subset of a backward arithmetic compact subset of an asymmetric metric space $(X, d)$ is backward arithmetic compact.

Proof. The proof is the same as in the previous theorem.

\section{R E F E R E N C E S}

1. G. E. Albert: A note on quasi metric spaces. Bull. Amer. Math. Soc. 47 (1941), 479-482.

2. J. Antoni and T. ŠAlÁt: On the A-continuity of real functions. Acta Math. Univ. Comenian. 39 (1980) 159-164.

3. A. S. Babu, T. Dosenovic, M. M. Ali, S. Radenovic and K. P. R. RaO: Some presic type results in b-dislocated metric spaces. Constr. Math. Anal. 2(1) (2019), 40-48.

4. H. ÇAKalli: A variation on arithmetic continuity. Bol. Soc. Paran. Mat. 35(3) (2017), 195-202; Corrigendum: Bol. Soc. Paran. Mat. 37(2) (2019), 179-181.

5. H. ÇaKalli: On G-continuity. Comput. Math. Appl. 61(2011), 313-318.

6. H. ÇAKAlLI and A. SönmeZ: Slowly oscillating continuity in abstract metric spaces. Filomat 27(5) (2013), 925-930.

7. H. ÇAKALLI: Upward and downward statistical continuities. Filomat 29(10) (2015), 2265-2273.

8. I. Chenchiah, M. O. Rieger and J. Zimmer: Gradient flows in asymmetric metric spaces, Nonlinear Anal. 71 (2009), 5820-5834.

9. S. Cobzaş: Functional Analysis in Asymmetric Normed Spaces. Frontiers in Mathematics, Springer, Basel, 2013.

10. J. Collins and J. Zimmer: An asymmetric Arzela-Ascoli theorem. Topology Appl. 154 (2007) 2312-2322.

11. J. Connor and K. G. Grosse-Erdmann: Sequential definition of continuity for real functions. Rocky Mountain J. Math. 33(1) (2003), 93-121.

12. Z. Kadelburg and S. RAdenovic: Notes on some recent papers concerning Fcontractions in b-metric spaces. Constr. Math. Anal. 1(2) (2018), 108-112.

13. J. C. Kelly: Bitopological spaces. Proc. Lond. Math. Soc. 13 (1963), 71-89.

14. H. P. A. KünZI: A note on sequentially compact pseudometric spaces. Monatsh. Math. 95 (1983), 219-220.

15. A. Mennucci: On asymmetric distances. Anal. Geom. Metr. Spaces 1 (2013), 200231.

16. I. L. Reilly, P. V. Subrahmanyam and M. K. Vamanamurthy: Cauchy sequences in quasi-pseudo-metric spaces. Monatsh. Math. 93 (1982), 127-140.

17. W. H. Ruckle: Arithmetical summability. J. Math. Anal. Appl. 396 (2012), 741-748.

18. W. A. Wilson: On quasi-metric spaces. Amer. J. Math. 53 (1931), 675-684.

19. T. YAYING and B. HAZARIKA: On arithmetical summability and multiplier sequences. Nat. Acad. Sci. Lett. 40(1) (2017), 43-46. 
20. T. YAYing and B. HazArika: On arithmetic continuity. Bol. Soc. Paran. Mat. 35(1) (2017), 139-145.

21. T. YAYING, B. HAZARIKA and H. ÇAKAlLI: New results in quasi cone metric spaces. J. Math. Comp. Sci. 16(3) (2016), 435-444.

22. T. YAYING and B. HAZARIKA: On arithmetic continuity in metric spaces. Afr. Mat. 28 (2017), 985-989.

23. T. YAYIng and B. HazARIKA: Lacunary arithmetic convergence. Proc. Jangjeon Math. Soc. 21(3) (2018), 507-513.

Taja Yaying

Department of Mathematics

Dera Natung Govt. College

Itanagar 791009

Arunachal Pradesh, India

tajayaying20@gmail.com

Bipan Hazarika

Department of Mathematics,

Gauhati University

Guwahati 781 014, Assam, India

bh_rgu@yahoo.co.in

Syed Abdul Mohiuddine

Operator Theory and Applications Research Group

Department of Mathematics

Faculty of Science

King Abdulaziz University

Jeddah 21589, Saudi Arabia

and

Department of General Required Courses, Mathematics

Faculty of Applied Studies

King Abdulaziz University

Jeddah 21589, Saudi Arabia

mohiuddine@gmail.com 